\title{
BehaviorDEPOT: a tool for automated behavior classification and analysis in rodents
}

2 Christopher J Gabriel ${ }^{1,2}$, Zachary Zeidler*2, Benita Jin*2,3, Changliang Guo ${ }^{4}$, Anna Wu ${ }^{2}$, Molly Delaney ${ }^{5}$, 3 Jovian Cheung ${ }^{5}$, Lauren E. DiFazio ${ }^{6}$, Melissa J. Sharpe ${ }^{6}$, Daniel Aharoni ${ }^{4}$, Scott A. Wilke , and Laura A. 4 DeNardo ${ }^{2, \#}$

5 *equal contribution

Author Affiliations: ${ }^{1}$ UCLA Neuroscience Interdepartmental Program, ${ }^{2}$ Department of Physiology,

$8{ }^{3}$ UCLA Molecular, Cellular, and Integrative Physiology Program, ${ }^{4}$ Department of Neurology,

$9{ }^{5}$ Department of Pyschiatry, ${ }^{6}$ Department of Psychology, University of California Los Angeles

${ }^{\#}$ Correspondence: Dr. Laura DeNardo, 10833 Le Conte Ave, Center for Health Sciences, David Geffen

School of Medicine at UCLA, Los Angeles, CA 90095 ldenardo@ucla.edu

\section{Abstract}

15 Quantitative descriptions of animal behavior are essential to understand the underlying neural substrates.

16 Many behavioral analyses are performed by hand or with expensive and inflexible commercial software

17 that often fail on animals with attached head implants, such as those used for in vivo optogenetics and

18 calcium imaging. With the development of machine learning algorithms that can estimate animal

19 positions across time and space, it is becoming easier for users with no prior coding experience to perform

20 automated animal tracking in behavioral video recordings. Yet classifying discrete behaviors based on

21 positional tracking data remains a significant challenge. To achieve this, we must start with reliable

22 ground truth definitions of behavior, a process that is hindered by unreliable human annotations. To

23 overcome these barriers, we developed BehaviorDEPOT (DEcoding behavior based on POsitional

24 Tracking), a MATLAB-based application comprising six independent modules and a graphical user

25 interface. In the Analysis Module we provide hard-coded classifiers for freezing and rearing. Optionally

26 applied spatiotemporal filters allow users to analyze behaviors in varied experimental designs (e.g. cued

27 tasks or optogenetic manipulations). Even inexperienced users can generate organized behavioral data

28 arrays that can be seamlessly aligned with neurophysiological recordings for detailed analyses of the

29 neural substrates. Four additional modules create an easy-to-use pipeline for establishing reliable ground-

30 truth definitions of behaviors as well as custom behavioral classifiers. Finally, our Experiment Module

31 runs fear conditioning experiments using an Arduino-based design that interfaces with commercial 
32 hardware and significantly reduces associated costs. We demonstrate the utility and flexibility of

33 BehaviorDEPOT in widely used behavioral assays including fear conditioning, avoidance, and decision-

34 making tasks. We also demonstrate the robustness of the BehaviorDEPOT freezing classifier across

35 multiple camera types and in mice and rats wearing optogenetic patch cables and head-mounted

36 Miniscopes. BehaviorDEPOT provides a simple, flexible, automated pipeline to move from pose tracking

37 to reliably quantifying a wide variety of task-relevant behaviors.

Introduction

40 A central goal of neuroscience is to discover relationships between neural activity and behavior. Discrete

41 rodent behaviors represent outward manifestations of cognitive and emotional processes. It is critical to

42 classify such behaviors rapidly and reliably with high spatiotemporal precision. The recent explosion of

43 techniques for manipulating or recording activity in neural circuits is advancing behavioral neuroscience

44 at an unprecedented pace ${ }^{1}$. However, a major bottleneck has been aligning these data with coincident

45 behavior, especially for laboratories without established expertise in this area. Much of this work has been

46 done manually, but automated detection of freely moving animal behaviors is faster, expands the

47 parameter space that can be explored, and removes human biases. The standardization promised by such

48 methods also enhances the rigor and reproducibility of results across research groups. However, these

49 analyses can be technically challenging for researchers to develop in-house.

50 Recently, behavioral neuroscientists have embraced machine learning algorithms for tracking

51 animal positions across time and space $^{2}$. Free and easy-to-use software packages such as DeepLabCut

52 (DLC) allow even inexperienced users to train deep neural networks that automatically track user-defined

53 points on an animal's body ${ }^{3}$. The static arrangement of those points at any moment represents a pose. The

54 challenge then is to classify temporal pose sequences as discrete behaviors that represent useful

55 information about the cognitive or emotional state of the animal. This is a major challenge for two main

56 reasons: 1) developing automated classifiers typically requires advanced programming skills and 2) 
57 developing robust classifiers requires accurate ground-truth definitions of behaviors, but human 58 annotations are error-prone and unreliable.

Quantifying animal behavior can be complex and technically challenging. Some behaviors can be defined exclusively by the animal's pose, such as during freezing in response to fearful stimuli. Other common assays, like the T-maze, elevated plus maze, and open field test, utilize spatiotemporal features to define behavior. Many labs currently score behaviors manually, a time-consuming, laborious, and error-prone process. Others use commercially available software that can be prohibitively expensive and fail when used with common headgear for in vivo neurophysiology. Moreover, manual scoring and commercial software typically only report when behavior was present, providing a limited view of the animal's behavior. Robustly analyzing spatial and temporal information can enhance behavioral assessment by providing important layers of information about the animal's emotional and physical status.

To overcome these challenges, we developed an open-source software package for behavior DEcoding based on POsitional Tracking (BehaviorDEPOT) that is robust, flexible and easy to use.

72 its graphical user interface (GUI), no coding experience is required to use BehaviorDEPOT, but

73 experienced users can tailor the software to their liking. The Analysis Module imports behavior videos

74 and accompanying pose tracking data and classifies behavior on a frame-by-frame basis. It includes hard-

75 coded classifiers for freezing, rearing, moving, and jumping. With optionally applied spatiotemporal

76 filters, it can perform automated behavioral analysis for any experimental design (e.g., cued tasks or

77 optogenetics experiments). Vectorized data is saved in MATLAB data arrays that facilitate additional

78 user-defined automated analyses. The Analysis Module also features customizable visualization tools that

79 integrate behavioral classification with spatiotemporal trajectory mapping. The Experiment Module can

80 run fear conditioning experiments using an Arduino-based protocol that is compatible with commercially

81 available shockers, arenas, and lasers for optogenetics. 
83 manner that requires no programming experience. A major hurdle in developing classifiers is settling on a

84 ground truth definition of the behavior of interest. It is difficult, particularly for unskilled raters, to

85 annotate hours of behavior videos without introducing errors or having annotations diverge amongst

86 individuals and across labs. However, several skilled raters, focusing on establishing ground-truth

87 definitions can establish behaviors which can then be more reliably annotated by automated classifiers.

88 The Inter-Rater Module simplifies this process by performing automated comparisons of manual

89 annotations from multiple human raters. Taking in frame-by-frame human annotations, it calculates the

90 level of inter-rater agreement and highlights points of disagreement so the raters can refine their

91 annotations until they converge maximally. The Inter-Rater Module can function as an independent unit

92 and can thus support classifier development with any application. The additional modules help users

93 perform parameter exploration, optimization, and validation of new classifiers (Figure 1).

95 classification and spatiotemporal analysis of commonly studied behaviors. We demonstrate

96 BehaviorDEPOT's utility with three frequently used behavioral assays, yet it is highly customizable,

97 assisting users to generate new classifiers with minimal coding involved. Other open-source software

98 packages that transform animal poses into discrete behaviors focus on social behaviors or behavioral

99 syllables that are not necessarily associated with specific emotional or motivational states (MARS ${ }^{4}$,

$100 \mathrm{SimBA}^{5}, \mathrm{MoSeq}^{6}, \mathrm{~B}-\mathrm{SOiD}^{7}$, and $\mathrm{DANNCE}^{8}$ ). To our knowledge, BehaviorDEPOT provides the only

101 open-source pose tracking-based freezing classifier that operates via a user-friendly GUI. Compared to

102 existing open-source freezing detection software ${ }^{9}$, BehaviorDEPOT can both implement and analyze fear

103 conditioning experiments. BehaviorDEPOT can perform batched data analyses and has the unique ability

104 to integrate behavior classification with the spatiotemporal trajectory of the animal. This facilitates

105 analysis of assays in which the experimenter may want to quantify not only how much an animal

106 performs a particular behavior overall, but where and when it does so. 


\section{Results}

109 BehaviorDEPOT functions as a MATLAB application or as a standalone executable file. Through its

110 GUI, users can interact with six independent modules (Figure 1, Figure S1). The Analysis Module

111 smooths pose tracking data and quantifies freezing in addition to a variety of defensive and ambulatory

112 behaviors including escape, running, and walking. Users can implement custom temporal and spatial

113 filters to analyze behaviors in varied experimental designs. The other four modules allow users to create

114 and optimize custom classifiers with little-to-no programming experience.

\section{Development of the BehaviorDEPOT Freezing Classifier}

117 BehaviorDEPOT combines a convolution-based smoothing operation with a low-pass filter to identify

118 periods of freezing (Figure 2A-D), defined as the absence of movement except for respiration. We began

119 by training two different deep neural networks in DeepLabCut. One is based on videos recorded with a

120 machine-learning quality camera at 50 frames per second (fps). This network tracks 8 points on the body

121 (Figure 2A). The second network is based on videos recorded with a standard webcam at 30fps. On the

122 webcam, lower sensor quality and lower frame rate produces more blur in the recorded images, so we

123 only tracked 4 body parts (nose, ears, tail-base; Figure 2). While it is possible to generate a single DLC

124 network that can estimate the positions of body parts with reasonable precision in both video types, we

125 found that more tailored networks produced lower jitter (i.e., small changes in point placement from

126 frame to frame) around the estimated points. Low jitter proved to be critical for creating the most robust

127 freezing classifier. Users can select the classifier that works best for their videos.

128 DLC produces a list of comma-separated values that contains framewise estimates of the $\mathrm{x}-\mathrm{y}$

129 coordinates for designated body parts as well as a likelihood statistic for each estimated point. We first

130 applied a threshold based on DLC likelihood $(\mathrm{p}<0.1)$ and performed a Hampel transformation ${ }^{10}$ to

131 remove outliers. Then, a LOWESS, local regression smoothing method was applied to the data ${ }^{11}$, and sub-

132 threshold tracking values were estimated using surrounding data and spline interpolation (Figure 2B). We

133 calculated statistics based on tracked points including linear velocity, acceleration, head angle, and 
134 angular velocity. After exploring the parameter space, we empirically determined that thresholding the

135 velocity of a weighted average of 3-6 body parts (depending on the frame rate of the video recording) and

136 the angle of head movements produced the best-performing freezing classifier (Figure 2C). We applied a

137 sliding window to produce a convolved freezing vector in which each value represents the number of

138 freezing frames visible in the window at a given frame. We then applied an adjustable count threshold to

139 convert the convolved freezing vector into the final binary freezing vector (Figure 2D).

140 We validated the performance of the freezing classifier by comparing it to manual scoring by

141 three trained raters. To ensure our classifiers were robust, our validation data sets included $\sim 30,000$

142 frames from 4-6 videos (at least 6000 frames per video) that were recorded in distinct behavioral

143 chambers under varied lighting conditions. Human ratings were indistinguishable from classifier

144 performance (Figure 2E,F). Accuracy of the freezing classifier was estimated based on classifier precision

145 (number of true positive frames / sum of true positive and false positive frames), recall (number of true

146 positive frames / sum of true positive and false negative frames), F1 score (two times the product of

147 precision and recall / sum of precision and recall) and specificity (number of true negative frames / sum of

148 true negative and false positive frames). Precision and recall quantify the positive predictive value of the

149 classifier against the tendency to produce false positive or false negative errors, respectively. The F1

150 score, the harmonic mean of the precision and recall, is useful as a summary statistic of overall classifier

151 performance. Specificity quantifies the classifier's ability to accurately label true negative values and

152 helps ensure that the classifier is capturing data from only a single annotated behavior. The high values in

153 all categories (Figure 2E,F) indicated that freezing classifiers based on webcams and machine learning

154 quality cameras both had excellent performance.

\section{The Analysis Module}

157 The Analysis Module imports videos and accompanying pose tracking data, smooths the data using a

158 user-specified method, and calculates movement statistics based on tracked points (e.g., linear velocity,

159 angular velocity, acceleration). It then analyzes behavior in a framewise manner by implementing custom 
160 classifiers that threshold movement and position statistics in unique combinations. In the GUI, users

161 indicate if they want to analyze behaviors during particular time windows or within regions of interest

162 (ROI), and whether they plan to do batched analyses. Depending on their choices, users are led through a

163 series of windows in which they indicate the body parts that they tracked, ROI location(s), and when time

164 windows of interest occurred. The Analysis Module analyzes behavior according to the user-defined

165 specifications and saves the data in a set of MATLAB structures. The 'Params' structure stores the

166 parameters of video recordings, smoothing, and arena metrics (e.g., ROI size and location). 'Tracking'

167 organizes and stores pose data from DLC. 'Metrics' stores calculated movement and position statistics

168 based on tracked points including linear velocity, angular velocity, and acceleration (Figure 2G).

169 'Behavior' stores bout-wise and vectorized representations of classified behaviors both in and out of user-

170 defined spatiotemporal filters. Organizing the data this way facilitates additional automated analyses of

171 the user's choosing. Users can track any points they like, though they may have to adjust and validate the

172 behavior thresholds using our Optimization and Validation Modules, described in upcoming sections.

173 The Analysis Module also generates a series of graphical data representations. Trajectory maps

174 show when an animal was in a particular location and where behaviors occurred. Bout maps indicate

175 when behaviors occurred and for how long. Additional graphs tell the user how much a particular

176 behavior occurred during presentations of a cue or optogenetic stimulation period. These visual

177 representations help users understand behavioral phenotypes in great spatiotemporal detail and can inform

178 development of further custom analyses using the data arrays that the Analysis Module generates.

\section{Use Case 1: Optogenetics}

181 In commercial freezing detection software, algorithms often fail when a rodent is wearing a patch cord for

182 routine optogenetics experiments. We set out to validate BehaviorDEPOT's utility in an optogenetics

183 experiment. The medial prefrontal cortex (mPFC) plays a well-established role in fear memory

184 retrieval $1^{12,13}$, extinction ${ }^{14,15}$, and generalization ${ }^{14,16,17}$. We performed an experiment to examine the role of

$185 \mathrm{mPFC}$ in contextual fear memory generalization. While silencing mPFC subregions can promote fear 
memory generalization in remote memory ${ }^{16,18}$, less is known about its role in recent memory

187 generalization. We used an adeno-associated virus (AAV) to express the soma-targeted neuronal silencer

188 stGtACR $2^{19}$ bilaterally in the mPFC and implanted optogenetic cannula directly above the AAV injection

189 sites (Figure 3A, Figure S2). We performed contextual fear conditioning (CFC) and then tested animals'

190 fear memory 24 hours later by recording their behavior in both the fearful context and in a novel

191 environment that was not previously paired with shocks (Figure 3B). We estimated the animals' position

192 on each frame using an optogenetics-specific DLC network that tracked 9 points on the animal, including

193 the fiber-optic cannula (Figure 3C). We then analyzed freezing levels with BehaviorDEPOT. Fear

194 conditioned mice readily froze following shocks during CFC, while non-shocked controls did not (Figure

195 3D). Silencing mPFC in previously shocked animals significantly enhanced freezing in the novel context

196 but did not affect freezing in the fearful context (Figure 3E,F). mPFC silencing thus produced a

197 significant decrease in the discrimination index in fear conditioned mice (Figure 3G), indicating that

198 mPFC plays a key role in the specificity of recent fear memories. In all analyses, BehaviorDEPOT

199 performance was indistinguishable from a highly trained human rater (Figure $3 \mathrm{E}-\mathrm{G}$ ) indicating that the

200 combination of the optogenetics-specific DLC model and BehaviorDEPOT achieves robust and accurate

201 freezing detection.

\section{Use Case 2: $\mathrm{Ca}^{2+}$ imaging with Miniscopes during signaled avoidance}

204 As new open-source tools for neurophysiology become available, increasing numbers of labs are

205 performing simultaneous neurophysiological and behavioral recordings to understand the neural

206 mechanisms of complex behaviors. Recent advances in miniature head-mounted microscopes now allow

207 us to image the activity of hundreds of neurons simultaneously in freely moving animals ${ }^{20-22}$. These

208 miniscopes pair with genetically encoded $\mathrm{Ca}^{2+}$ indicators ${ }^{23}$ that can be targeted to genetically,

209 anatomically, or behaviorally-defined neuronal populations, and GRIN lenses ${ }^{24}$ that can be targeted

210 anywhere in the brain. With these tools in hand, we are now able to discover how the encoding of

211 complex cognitive and emotional behaviors maps onto specific cell types across the brain. By recording 
212 the activity of hundreds of neurons simultaneously, we can also begin to understand the population codes

213 that produce complex, freely moving behaviors ${ }^{25,26}$. To do so, however, we need improved methods that

214 allow us to rapidly and accurately quantify freely moving behaviors with reference to salient

215 environmental stimuli and to align these detailed behavioral measurements with neurophysiological

216 recordings. BehaviorDEPOT directly addresses these deficits by automatically integrating and

217 quantifying spatial information (e.g animal location), temporal information (e.g. onset of conditioned

218 cues), and the time and location at which discrete behaviors occurred. Stored in vectorized data structures

219 in a framewise manner, these detailed behavioral data can be instantly aligned with physiological

220 recordings.

221 Here we demonstrate the utility of BehaviorDEPOT for aligning behavioral measurements with

$222 \mathrm{Ca}^{2+}$ signals during a signaled avoidance task that has temporally and spatially salient features. In our

223 task, which is a refinement of platform mediated avoidance ${ }^{27}$, a fear conditioned tone prompts mice to

224 navigate to a safety platform that protects them from receiving a footshock. During a behavioral session,

225 animals toggle between conditioned freezing and environmental exploration in a manner that is

226 modulated by temporal (e.g. conditioned tones) and spatial (e.g. safety platform) factors. In this task, the

227 medial prefrontal cortex acts through downstream target regions to determine whether an animal

228 approaches or avoids environmental $\operatorname{stimuli~}^{28}$. To determine how ensembles of prefrontal neurons drive

229 behavior during PMA, we recorded the activity of hundreds of mPFC neurons in freely behaving animals

230 using head-mounted microendoscopes (UCLA Miniscopes ${ }^{21,22}$ ) while simultaneously recording behavior

231 using a new open-source USB camera, the UCLA MiniCAM.

232 The MiniCAM is an open-source behavioral imaging platform that natively integrates and

233 synchronizes with open-source UCLA Miniscope hardware and software (Figure 4A). It is composed of

234 an M12 optical lens mount, a custom printed circuit board housing a CMOS image senor and supporting

235 electronics, an LED illumination ring, and a 3D printed case. The MiniCAM is powered and

236 communicates over a single coaxial cable that can be up to 15 meters long. The coaxial cable connects to

237 a Miniscope data acquisition board (DAQ) which then connects over USB3 to a host computer. A range 
of commercial M12 lenses can be used to select the view angle of the camera system. The image sensor used is a 5MP CMOS image sensor (MT9P031I12STM-DP, ON Semiconductor) with $2592 \times 1944$ pixel resolution and a full resolution frame rate of approximately 14FPS. For this application, the MiniCAM's

241 pixels were binned and cropped to achieve 1024X768 pixels at approximately 50FPS. The optional LED

242 illumination ring uses 16 adjustable red LEDs (LTST-C190KRKT, Lite-On Inc., 639nm peak

243 wavelength) for illumination in dark environments (Figure 4A). We trained a separate DLC network for

244 videos of animals wearing Miniscopes recorded with MiniCAMs. Our network tracked 8 points on the

245 body (ears, nose, midback, hips, tailbase, and tail) and the Miniscope itself (Figure 4B). Using the output

246 of this network, BehaviorDEPOT classified freezing with high fidelity in a manner that was

247 indistinguishable from highly trained users (Figure 4C).

248 We used BehaviorDEPOT to analyze behavior during PMA so we could align it to the underlying

249 neural activity. In this task, animals hear 3 baseline tones $(4 \mathrm{kHz}, 30 \mathrm{~s})$ followed by 9 tones that co-

250 terminate with a mild foot shock $(0.15 \mathrm{~mA}, 2 \mathrm{~s})$. An acrylic platform occupies $25 \%$ of the electrified grid

251 floor, providing a safe place to avoid the shock. The following day, animals are presented with 6

252 unreinforced tones, and we measure the resulting avoidance and freezing responses (Figure 4D). The

253 Analysis Module automatically produces maps that make it quick and easy to assess the spatiotemporal

254 characteristics of rodent behavior. In our representative example, the color-coded trajectory and freezing

255 locations (denoted as black squares) converge on the platform at the end of the session, indicating the

256 mouse indeed learned to avoid shocks by entering the platform (Figure 4E). The vectorized session data

257 from the Experiment Module (e.g. tone times, shock times) and behavioral data from the Analysis module

258 can be easily integrated for additional analyses. We visualized the temporal dynamics of learning by

259 overlaying fraction time on the platform and freezing on the tone periods (Figure 4F). We also used

260 BehaviorDEPOT to produce summary data, showing that mice readily learned and remembered the cue-

261 avoidance association as expected (Figure 4G).

262 During a retrieval session, we performed simultaneous recordings of neural activity using a

263 UCLA Miniscope and behavior using a UCLA MiniCAM (Figure 4H). Using MIN1PIPE ${ }^{29}$, we extracted 
264 and processed neural signals from $513 \mathrm{mPFC}$ neurons across 3 mice. We then determined whether

265 individual neurons encoded specific behaviors that we had quantified using BehaviorDEPOT. We

266 computed a receiver operator characteristic (ROC) curve that measures a neuron's stimulus detection

267 strength over a range of thresholds (Figure 4I,J). We identified numerous neurons that were modulated by

268 freezing and avoidance on the safety platform. These neurons were organized in a salt and pepper manner

269 in mPFC (Figure 4K). Nearly half of all neurons that exhibited task relevant behavior and were specific

270 for either freezing or avoiding on the platform, or the combination of both (Figure 4L).

\section{The Inter-Rater Module}

273 A major challenge in behavioral neuroscience is establishing reliable ground truth definitions of behavior.

274 Even when using automated behavioral classification systems like BehaviorDEPOT, it is critical to have

275 reliable behavioral annotations from multiple human raters to validate the performance of the classifier.

276 The Inter-Rater Module helps users establish reliable ground truth behavioral definitions in two ways.

277 First, the Inter-Rater Module imports manual behavioral scoring from human raters and automatically

278 compares the scores on a frame-by-frame basis. It calculates the level of agreement and reports points of

279 disagreement both numerically and graphically. In response to the initial output of the Inter-Rater

280 Module, human raters can discuss the points of disagreement and modify their manual ratings

281 accordingly. The automated features of the Inter-Rater Module make it fast and easy to perform iterative

282 comparisons of manual annotations, interleaved with human updates, until a satisfactory level of

283 agreement is achieved.

284 The Inter-Rater Module prompts the user to select a file directory containing one or more human 285 annotation files and automatically imports the data into MATLAB. The user then selects the annotations

286 to include in the analysis, the reference dataset, and the behavior of interest. After all parameters are set,

287 the module compares each set of annotations to the reference, scoring the annotations frame-by-frame as

288 true positive, true negative, false positive, or false negative for each rater. These values are first used to

289 calculate percent overlap and percent error between all raters. Precision, recall, specificity, and F1 score 
290 are calculated and reported for each rater relative to the chosen reference. Additionally, visualizations of

291 frame-by-frame percent agreement and user-by-user disagreement are automatically generated to assist

292 identifying areas of conflict between users (Figure 5A). We developed the BehaviorDEPOT freezing

293 classifier based on the averaged ratings of three highly trained raters. Here, the Inter-Rater Module

294 demonstrates visualizations of agreement levels for three highly trained raters and a novice freezing rater

295 (Figure 5B). By illustrating frames with high level of disagreement, the Inter-Rater Module can help labs

296 train new human annotators while building ground truth definitions for new classifiers.

298 The Data Exploration Module

299 The Data Exploration Module is designed to help refine existing classifiers and to generate entirely new

300 ones. When developing behavior classifiers, it is advantageous to explore the parameter space of

301 positional tracking data and identify the metrics that track most closely with a behavior of interest. The

302 Data Exploration Module allows users to explore the metrics that BehaviorDEPOT calculates based on

303 tracked points and determine whether chosen metrics separate behavior data accurately, using human

304 labels as a reference. The chosen metrics are used to generate a generalized linear model (GLM) that

305 estimates the likelihood that the behavior is present in a frame, given different values of the selected

306 metrics (Figure 6A). This module allows users to easily identify and compare metrics that can form the

307 basis of new classifiers or enhance the performance of existing ones.

308 The Data Exploration Module prompts the user to select a directory containing analyzed data and

309 a human rater file, and the data is then automatically imported into MATLAB. Users can select two of the

310 metrics from the analyzed file (e.g., head velocity, tail angular velocity, etc.) and a behavior label from

311 the human annotations file. The module then creates two data distributions: one containing video frames

312 labeled with the chosen behavior and a second consisting of the remaining video frames (Figure 6B). The

313 module will randomly downsample the larger set to ensure that each distribution contains equal numbers

314 of frames. The module then performs a series of analyses to quantify how reliably chosen metrics align

315 with the behavior of interest. Histograms and boxplot representations of metric values for behavior- 
316 containing and non-behavior-containing frames help users identify metric values that reliably segregate

317 with frames containing a behavior of interest (Figure 6C). Chosen metrics then form the basis of a GLM

318 that predicts the likelihood that a frame captures the chosen behavior based on different values of each of

319 the chosen metrics (Figure 6D). Metrics that are well-suited for behavior classification contrast with

320 metrics on frames that do not contain the behavior and have a low standard deviation within the behavior

321 set. Distributions of useful metrics also tend to differ substantially from the total set of frames, especially

322 when compared to frames that do not contain the behavior. The GLM predictions are useful for

323 determining which of the selected metrics best predict the behavior and whether they enhance the

324 predictive value when combined.

\section{The Classifier Optimization Module}

327 When developing a new classifier or optimizing our freezing classifier for use with externally generated

328 data, BehaviorDEPOT's Classifier Optimization Module allows user to rapidly assess how changes in

329 classifier parameters (i.e. thresholded positional metrics) affect classifier performance (Figure 7A). To

330 ensure that BehaviorDEPOT performs robustly across laboratories, we used the Classifier Optimization

331 Module to adjust our freezing classifier for analysis of behavioral videos of rats and mice, respectively,

332 that were recorded in two external laboratories using their own acquisition hardware and software (Figure

$3337 \mathrm{~B}_{1,2}$ ). The Classifier Optimization Module takes in a BehaviorDEPOT-analyzed tracking file and

334 associated human annotations and prompts the user to select up to two parameters from their chosen

335 classifier. The user can then input a list of parameter values to test on the classifier. The module then runs

336 the classifier using every combination of test values and calculates the performance (precision, recall, F1

337 score, and specificity) for each parameter combination. These values are stored in a MATLAB data array

338 and automatically visualized using easy-to-read heatmaps. Importantly, the freezing classifier parameters

339 that worked well for the data collected in our laboratory (change in head angle and head velocity) also

340 worked well for the data collected in other labs. We used the Classifier Optimization Module to identify

341 the combination of thresholds for two metrics (averaged head/rear back velocity and change in head 
342 angle, Figure $7 \mathrm{C}_{1,2}$ ) and the combination of window width and count threshold in the convolution

343 algorithm (Figure $7 \mathrm{D}_{1,2}$ ) that produced maximal F1 scores. This module helps users optimize parameter

344 values for individual classifiers and can be used in conjunction with the Data Exploration Module to build 345 and test new behavior classifiers in a way that requires no prior coding experience.

\section{The Validation Module}

348 The Validation Module is designed to quickly assess a classifier's predictive quality. To ensure that

349 behavioral classifications are robust to different video recording conditions, the Validation Module

350 compares classifier output to trained human raters across frames from multiple videos. The user will

351 initially be prompted to indicate which classifier to evaluate and select a directory containing behavior

352 videos and accompanying BehaviorDEPOT-analyzed tracking data. For each video, the module will

353 categorize each frame as true positive, false positive, true negative, or false negative, using the human

354 data as a reference. Precision, recall, specificity, and F1 score are then calculated and visualized for each

355 video. These statistics are also reported for the total video set by concatenating all data and recalculating

356 performance (Figure 7E). We used this data to validate the performance of the BehaviorDEPOT freezing

357 classifier on the rat (Figure 7F) and mouse (Figure 7G) videos we acquired from external laboratories.

Automated Quantification of Decision-Making Behaviors

360 BehaviorDEPOT can be flexibly adapted for behaviors beyond the realm of conditioned fear. Many labs

361 study contingency-based, cost-benefit decision-making in mazes that involve choice points. For example,

362 effort-based decision-making involves weighing whether it is worth exerting greater effort for a more

363 valuable outcome. A common rodent assay for effort-based decision-making is the barrier T-maze task in

364 which animals choose whether to climb over a barrier for a large reward (high effort, high value reward;

365 HE/HVR) vs. taking an unimpeded path for a smaller reward (low effort, low value reward; LE/LVR) ${ }^{30}$.

366 Well trained animals typically prefer HE/HVR choices, taking a direct route over the barrier with

367 stereotyped trajectories. However, when reward or effort contingencies are adjusted animals demonstrate 
vicarious trial and error (VTE), thought to be a marker of deliberative (rather than habitual) decisionmaking. During VTE trials, animals may pause, look back-and-forth, or reverse an initial choice ${ }^{31}$.

370 Several groups have identified neural signatures of VTE in hippocampus, striatum and prefrontal cortex

371 suggesting that animals may be playing out potential choices (i.e. vicariously) based on an internal model

372 of maze contingencies ${ }^{31}$. Simple, flexible, and automated analysis tools for detecting VTE and aligning

373 this behavior with neural data would significantly enhance our understanding of deliberative decision-

374 making.

375 We used BehaviorDEPOT to automatically detect VTE in a barrier T-maze task and to report the 376 ultimate choice animals made during each effort-based decision-making trial. First, we trained a new

377 DLC network to track mice as they navigated the T-maze. In one version of the task, mice decided

378 whether to turn left to collect a small reward or to turn right and climb a wire mesh barrier for a large

379 reward. We then changed the effort contingency by adding a second barrier to equalize effort across

380 choices (Figure 8A,B). We used the BehaviorDEPOT Analysis Module to perform automated analysis of

381 the task. We defined 8 regions of interest (Approach, Choice, Left Effort, Left Reward, Left Food Cup,

382 Right Effort, Right Reward, and Right Food Cup). We then used the stored tracking data to automatically

383 detect trials, which we defined as the first frame when the animal entered the approach zone until the first

384 frame when the animal entered the reward zone, and to report the outcome of each trial (left choice or 385 right choice) (Figure 8C,D).

To develop a VTE classifier, we used the head angle data stored in the BehaviorDEPOT 'Metrics'

387 data array. For each trial, we analyzed the head angles when the mouse was in the choice zone and used

388 these values to determine the number of head turns per trial. Manually annotated VTE trials tended to

389 have 1 or more head turns, while non-VTE trials tended to have 0 head turns, so we defined VTE trials as

390 having 1 or more head turns in the choice zone (Figure 8E). We then used our BehaviorDEPOT VTE

391 classifier to detect the fraction of trials with VTE in T-maze sessions with 1 vs. 2 barriers, finding a

392 significant increase in the occurrence of VTE trials when a second barrier was added. Importantly, the

393 BehaviorDEPOT performance was indistinguishable from a human rater (Figure 8E). These analyses 
394 highlight the utility of the robust repository of movement statistics that is automatically generated in

395 BehaviorDEPOT. By calculating and storing information including velocity, angular velocity, head angle,

396 and acceleration in a framewise manner, BehaviorDEPOT allows users to design automated analysis

397 pipelines for a wide range of commonly studied cognitive tasks.

\section{Discussion}

BehaviorDEPOT extends the utility of existing popular tools for markerless point tracking (e.g.

$401 \mathrm{DLC}^{3}$ and $\mathrm{LEAP}^{32}$ ) to create an open source, flexible, reliable, and automated behavioral analysis

402 pipeline. As optogenetics, miniscopes, and open-source technologies for extracellular recording become

403 available to increasing numbers of labs, users with little coding experience need easy-to-use programs

404 that can align detailed behavioral analyses with neurophysiological recordings and manipulations.

405 BehaviorDEPOT offers a graphical interface-based pipeline for running experiments and classifying

406 behaviors of interest with reference to spatial and/or temporal (e.g., tone times, optogenetic stimulation

407 times) experimental considerations. Dedicated modules allow even inexperienced users to create, test, and

408 optimize behavioral classifiers of their choosing.

We highlight the immediate utility of BehaviorDEPOT for analyzing behavior in widely used

410 assays including contextual fear conditioning, active avoidance, and effort-based decision making. We

411 report the first DLC-based freezing classifier with a user-friendly GUI and validate our freezing classifier

412 in a variety of experimental designs with potential visual confounds: unoperated mice, mice and rats from

413 our lab and others wearing an optogenetic patch cable, and mice wearing Miniscopes. In this way, our

414 freezing classifier succeeds in scenarios in which commercially available alternatives often fail. To

415 support miniscope-based analyses, we also report the development of the UCLA Minicam, which

416 interfaces seamlessly with the BehaviorDEPOT to allow high quality tracking and automated behavior

417 analysis in mice wearing UCLA Miniscopes. Using the classifier optimization module, we achieve robust

418 freezing classification in videos of mice and rats recorded in external labs, highlighting the utility of

419 BehaviorDEPOT for analyzing data that has already been collected. Finally, we leveraged the arrays of 
420 movement statistics that BehaviorDEPOT automatically generates to define trials in a T-maze task, report

421 animal choice, and identify instances of VTE. These examples showcase BehaviorDEPOT as an easy-to-

422 use, general utility software that is applicable to a wide range of behavioral neuroscience experiments.

423 BehaviorDEPOT fills an increasingly apparent gap in current resources. We integrate markerless

424 point tracking with top-down recordings, enabling behavioral classification with two-dimensional spatial

425 precision in an interface that requires no coding experience. Existing open-source software has succeeded

426 in addressing other specific needs. For instance, ezTrack ${ }^{9}$ enables robust, Python-based freezing

427 classification without the need of point tracking, but is limited to side-view videos, which constrains

428 spatial analyses. $\mathrm{MoSeq}^{6}$ and $\mathrm{DANNCE}^{8}$ combine 3-dimensional video recordings with unsupervised

429 machine learning to identify subsecond behavioral motifs, the complexity and coding-requirements of

430 which may be beyond the scope of experimenters interested in more macro-level behaviors. Additional

431 classifiers utilize multi-animal tracking for analyzing social behaviors ${ }^{4,5}$ and grooming behaviors ${ }^{7,8}$. With

432 a well-validated freezing classifier and simple GUI that can run experiments and analyze, organize and

433 align data, BehaviorDEPOT will bridge the gap between pose-tracking algorithms and neurophysiological

434 recordings and manipulations for labs with any level of coding experience.

\section{References}

437 1. Luo, L., Callaway, E. M. \& Svoboda, K. Genetic Dissection of Neural Circuits: A Decade of $438 \quad$ Progress. Neuron (2018) doi:10.1016/j.neuron.2018.03.040.

439 2. Mathis, M. W. \& Mathis, A. Deep learning tools for the measurement of animal behavior in 440 neuroscience. Curr. Opin. Neurobiol. 60, 1-11 (2020).

441 3. Mathis, A. et al. DeepLabCut: markerless pose estimation of user-defined body parts with deep 442 learning. Nat. Neurosci. 21, (2018).

443 4. Segalin, C. et al. The Mouse Action Recognition System (MARS): a software pipeline for 444 automated analysis of social behaviors in mice. bioRxiv 2020.07.26.222299 (2020)

445 doi:10.1101/2020.07.26.222299. 
446 5. Nilsson, S. R. O. et al. Simple Behavioral Analysis (SimBA) - an open source toolkit for computer classification of complex social behaviors in experimental animals. bioRxiv 2020.04.19.049452 (2020) doi:10.1101/2020.04.19.049452.

6. Wiltschko, A. B. et al. Revealing the structure of pharmacobehavioral space through motion sequencing. Nat. Neurosci. 23, 1433-1443 (2020).

7. Hsu, A. I. \& Yttri, E. A. B-SOiD: An Open Source Unsupervised Algorithm for Discovery of

453 8. Dunn, T. W. et al. Geometric deep learning enables 3D kinematic profiling across species and 454 environments. Nat. Methods 18, 564-573 (2021).

455 9. Pennington, Z. T. et al. ezTrack: An open-source video analysis pipeline for the investigation of 456 animal behavior. Sci. Rep. 9, 1-11 (2019).

457 10. Hampel, F. R. The Influence Curve and its Role in Robust Estimation. J. Am. Stat. Assoc. 69, 383393 (1974).

459 11. Cleveland, W. S. LOWESS: A Program for Smoothing Scatterplots by Robust Locally Weighted Regression. Am. Stat. 35, 54 (1981).

461 12. Corcoran, K. A. \& Quirk, G. J. Activity in prelimbic cortex is necessary for the expression of learned, but not innate, fears. J. Neurosci. 27, 840-844 (2007).

463 13. Ledoux, J. E. Emotion circuits in the brain. Annu. Rev. Neurosci. 155-184 (2000).

464 14. Giustino, T. F. \& Maren, S. The Role of the Medial Prefrontal Cortex in the Conditioning and Extinction of Fear. Front. Behav. Neurosci. 9, 1-20 (2015).

466 15. Sierra-Mercado, D., Padilla-Coreano, N. \& Quirk, G. J. Dissociable Roles of Prelimbic and

16. Xu, W. \& Südhof, T. C. A neural circuit for memory specificity and generalization. Science. 339, $1290-1295$ (2013).

471 17. Pollack, G. A. et al. Cued fear memory generalization increases over time. Learn. Mem. 25, 298- 
473 18. Frankland, P. W., Bontempi, B., Talkton, L. E., Kaczmarek, L. \& Silva, A. J. The Involvement of the Anterior Cingulate Cortex in Remote Contextual Fear Memory. Science. 304, 881-883 (2004).

475 19. Mahn, M. et al. High-efficiency optogenetic silencing with soma-targeted anion-conducting 476 channelrhodopsins. Nat. Commun. 9, 4125 (2018).

477 20. Ghosh, K. K. et al. Miniaturized integration of a fluorescence microscope. Nat. Methods 8, 871$478 \quad 878(2011)$

479 21. Cai, D. J. et al. A shared neural ensemble links distinct contextual memories encoded close in $480 \quad$ time. Nature (2016) doi:10.1038/nature17955.

481 22. Shuman, T. et al. Breakdown of spatial coding and interneuron synchronization in epileptic mice. Nat. Neurosci. 23, 229-238 (2020).

23. Dana, H. et al. High-performance calcium sensors for imaging activity in neuronal populations and microcompartments. Nat. Methods (2019) doi:10.1038/s41592-019-0435-6.

24. Barretto, R. P. J., Messerschmidt, B. \& Schnitzer, M. J. In vivo fluorescence imaging with highresolution microlenses. Nat. Methods 6, 511-512 (2009).

487 25. Jercog, D. et al. Dynamical prefrontal population coding during defensive behaviours. Nature (2021) doi:10.1038/s41586-021-03726-6.

489 26. Stout, J. J. \& Griffin, A. L. Representations of On-Going Behavior and Future Actions During a Spatial Working Memory Task by a High Firing-Rate Population of Medial Prefrontal Cortex Neurons. Front. Behav. Neurosci. 14, 1-17 (2020).

492 27. Bravo-Rivera, C., Roman-Ortiz, C., Montesinos-Cartagena, M. \& Quirk, G. J. Persistent active avoidance correlates with activity in prelimbic cortex and ventral striatum. Front. Behav. Neurosci. (2015) doi:10.3389/fnbeh.2015.00184.

28. Diehl, M. M. et al. Divergent projections of the prelimbic cortex bidirectionally regulate active avoidance. Elife 9, 1-13 (2020).

29. Lu, J. et al. MIN1PIPE: A Miniscope 1-Photon-Based Calcium Imaging Signal Extraction 
Pipeline. Cell Rep. (2018) doi:10.1016/j.celrep.2018.05.062.

499 30. Bailey, M. R., Simpson, E. H. \& Balsam, P. D. Neural substrates underlying effort, time, and risk-

501 31. Redish, A. D. Vicarious trial and error. Nat. Rev. Neurosci. 17, 147-159 (2016).

502 32. Pereira, T. D. et al. Fast animal pose estimation using deep neural networks. Nat. Methods 16, $117-125$ (2019).

33. Shuman, T. et al. Breakdown of spatial coding and interneuron synchronization in epileptic mice. Nat. Neurosci. (2020) doi:10.1038/s41593-019-0559-0.

Author Contributions L.A.D., S.A.W., and C.J.G. conceived of experiments. C.J.G. and B.J. developed the freezing classifier. Z.Z. created MATLAB GUIs. C.J.G., B.J., Z.Z., and A.W. performed manual annotations of behavior. C.J.G. performed data acquisition and analysis of optogenetics experiments and Miniscope recordings. Z.Z. contributed to Miniscope data analysis. C.G. and D.A. designed and fabricated the UCLA MiniCAM and wrote accompanying software. M.J.S. and L.E.D. collected

512 behavioral data from rats. C.J.G., Z.Z., B.J., S.A.W., and L.A.D. wrote the paper. All authors read and 513 edited the paper.

515 Competing Interests The authors declare no competing interests.

517 Acknowledgements We thank A. Klein and N. Gogolla for contributing mouse behavioral videos for 518 analysis with BehaviorDEPOT. We thank J. Reichl for assistance with DLC network training. This work 519 was funded by NIH K01MH116264 (L.A.D.), Whitehall Foundation Research Grant (L.A.D), 520 Klingenstein-Simons Foundation Grant (L.A.D.), NARSAD Young Investigator Award (L.A.D.), NIH 521 T32MH073526 (B.J.), ARCS Pre-doctoral Fellowship (C.J.G.), NIMH K08 (S.A.W.).

\section{Methods}


525 Animals

526 Female and male C57B16/J mice (JAX Stock No. 000664) were group housed (2-5 per cage) and kept on

527 a $12 \mathrm{hr}$ light cycle. Following behavior conditioning, animals were individually housed until the memory

528 retrieval sessions. All animal procedures followed animal care guidelines approved by the University of

529 California, Los Angeles Chancellor's Animal Research Committee.

\section{Contextual fear conditioning}

532 Mice were handled for 5 days preceding the behavioral testing procedure. The conditioning chamber

533 consisted of an $18 \mathrm{~cm} \times 18 \mathrm{~cm} \times 30 \mathrm{~cm}$ cage with a grid floor wired to a scrambled shock generator

534 (Lafayette Instruments) surrounded by a custom-built acoustic chamber. The chamber was scented with

$53550 \%$ Windex. Mice were placed in the chamber and then after a 2-minute baseline period, received 5

$536 \quad 0.75 \mathrm{~mA}$ footshocks spaced 1 minute apart. Mice were removed 1 minute after the last shock. Non-

537 shocked control animals freely explored the conditioning chamber but never received any shocks. The

538 following day, mice were returned to the conditioning chamber and a novel context (different metal floor,

539 scented with $1 \%$ acetic acid), separated by a 1 -hour interval. Context presentation order on day 2 was

540 counterbalanced across mice.

\section{$542 \quad$ Platform-mediated avoidance}

543 PMA used the fear conditioning chamber described above, except $25 \%$ of the floor was covered with a

544 thin acrylic platform $(3.5 \times 4 \times 0.5$ inches $)$. During training, mice were presented with 3 baseline $30 \mathrm{~s} 4 \mathrm{kHz}$

545 tones (CS), followed by 9 presentations of the CS that co-terminated with a $2 \mathrm{~s}$ footshock $(0.13 \mathrm{~mA})$. The

546 following day, mice were presented with 6 CS in the absence of shocks. 
For T-maze experiments, mice were food deprived to $\sim 85 \%$ of their ad libitum initial weight (3-4 days) and habituated to handling and maze exploration. Reward pellets were chopped Reese's peanut butter chips ( $\sim 0.01 \mathrm{~g}$ each). One arm of the maze was designated as high value reward ('HVR', 3 pellets), the

552 other low value reward ('LVR', 1 pellet). Mice were trained to perform effort-reward decision-making via

553 a sequential process. Once mice learned to choose the HVR arm (>80\%), a $10 \mathrm{~cm}$ wire-mesh barrier was

554 inserted to block that arm. Training was complete when mice achieved $>70 \%$ success high effort/HVR

555 choices on 2 consecutive days. Forced trials were used to encourage sampling of the barrier arm during

556 training. Once mice achieved stable performance, a second barrier was inserted in the LVR arm to

557 equalize effort between choices.

Viruses

AAV1-syn-jGCaMP7f.WPRE (ItemID: 104488-AAV1) were purchased from Addgene and diluted to a working titer of $8.5 \times 10^{12} \mathrm{GC} / \mathrm{ml}$. and AAV1-CamKIIa-stGtACR2-FusionRed (ItemID: 105669-AAV1) were purchased from Addgene and diluted to a working titer of $9.5 \times 10^{11} \mathrm{GC} / \mathrm{ml}$.

\section{AAV injection with Optogenetic Cannula Implant}

565 Adult wildtype C57/B16 mice were anesthetized with isoflurane and secured to a stereotaxic frame. Mice

566 were placed on a heating blanket and artificial tears kept their eyes moist throughout the surgery. After

567 exposing the skull, we drilled a burr hole above mPFC in both hemispheres (AP+1.8, ML+/-0.3 from

568 bregma). A Hamilton syringe containing AAV-CaMKIIa-stGtACR2-WPRE was lowered into the burr

569 hole and $400 \mathrm{~nL}$ of AAV was pressure injected into each site (DV -2.25mm and $-2.50 \mathrm{~mm}$ from bregma) at

$570100 \mathrm{~nL} / \mathrm{min}$ using a microinjector (Kopf, 693A). The syringe was left in place for 10 minutes to ensure the

571 AAV did not spill out of the target region. After injecting the AAV, chronic fiber-optic cannula (0.37NA,

572 length $=2 \mathrm{~mm}$, diameter $=200 \mathrm{um}$ ) were implanted bilaterally above the injection site and secured to the

573 skull with Metabond (Parkell, S371, S396, S398). After recovery, animals were housed in a regular 12hr

574 light/dark cycle with food and water ad libitum. Carprofen $(5 \mathrm{mg} / \mathrm{kg})$ was administered both during 
575 surgery and for $2 \mathrm{~d}$ after surgery together with amoxicillin $(0.25 \mathrm{mg} / \mathrm{mL})$ in the drinking water for $7 \mathrm{~d}$ after

576 surgery.

\section{Miniscope Surgery and Baseplating}

579 For Miniscope recordings, all mice underwent two stereotaxic surgeries ${ }^{21,33}$. First, adult WT mice were

580 anesthetized with isoflurane and secured to a stereotaxic frame. Mice were placed on a heating blanket

581 and artificial tears kept their eyes moist throughout the surgery. After exposing the skull, a burr hole was

582 drilled above PL in the left hemisphere $(+1.85,-0.4,-2.3 \mathrm{~mm}$ from bregma). A Hamilton syringe

583 containing AAV1-Syn-jGCaMP7f-WPRE was lowered into the burr hole and 400nL of AAV was

584 pressure injected using a microinjector (Kopf, 693A). The syringe was left in place for 10 minutes to

585 ensure the AAV did not spill out of the target region and then the skin was sutured. After recovery,

586 animals were housed in a regular $12 \mathrm{hr}$ light/dark cycle with food and water ad libitum. Carprofen

$587(5 \mathrm{mg} / \mathrm{kg})$ was administered both during surgery and for $2 \mathrm{~d}$ after surgery together with amoxicillin $(0.25$

$588 \mathrm{mg} / \mathrm{mL}$ ) for $7 \mathrm{~d}$ after surgery. Two weeks later, mice underwent a GRIN lens implantation surgery. After

589 anesthetizing the animals with isoflurane (1-3\%) and securing them to the stereotaxic frame, the cortical

590 tissue above the targeted implant site was carefully aspirated using 27 gauge and 30-gauge blunt needles.

591 Buffered ACSF was constantly applied throughout the aspiration to prevent tissue desiccation. The

592 aspiration ceased after full termination of bleeding, at which point a GRIN lens ( $1 \mathrm{~mm}$ diameter, $4 \mathrm{~mm}$

593 length, Inscopix 1050-002176) was stereotaxically lowered to the targeted implant site (-2.0 mm

594 dorsoventral from skull surface relative to bregma). Cyanoacrylate glue was used to affix the lens to the

595 skull. Then, dental cement sealed and covered the exposed skull, and Kwik-Sil covered the exposed

596 GRIN lens. Carprofen $(5 \mathrm{mg} / \mathrm{kg})$ and dexamethasone $(0.2 \mathrm{mg} / \mathrm{kg})$ were administered during surgery and

597 for $7 \mathrm{~d}$ after surgery together with amoxicillin $(0.25 \mathrm{mg} / \mathrm{mL})$ in the drinking water. 2 weeks after

598 implantation, animals were anesthetized again with isoflurane (1-3\%) and a Miniscope attached to an

599 aluminum baseplate was placed on top of the GRIN lens. After searching the field of view for in-focus 
600 cells, the baseplate was cemented into place and the Miniscope was detached from the baseplate. A plastic

601 cap was locked into the baseplate to protect the implant from debris.

\section{Optogenetics}

604 Animals were habituated to the patch-cord for 3 days in advance of optogenetic stimulation. A patch-cord

605 was connected to the fiber-optic cannula and animals were allowed to explore a clean cage for 5 minutes.

606 On the testing day, optical stimulation through the fiber-optic connector was administered by delivering

607 light through a patch-cord connected to a 473-nm laser (SLOC, BL473T8-100FC). Stimulation was

608 delivered continuously with $2.5 \mathrm{~mW}$ power at the fiber tip.

\section{Miniscope Recordings}

611 Mice were handled and habituated to the weight of the microscope for 4 days before behavioral

612 acquisition. On the recording day, a V4 Miniscope was secured to the baseplate with a set screw and the

613 mice were allowed to acclimate in their home cage for 5 minutes. Imaging through the Miniscope took

614 place throughout the entire PMA training $(\sim 30 \mathrm{~min})$ and retrieval session the following day. Behavior was

615 simultaneously recorded with a UCLA MiniCAM.

\section{Behavior Video Recordings}

618 Behavioral videos were acquired using one of the following 3 setups:

619 1) 50fps using a Chameleon3 3.2 megapixel monochrome USB camera fitted with a Sony $1 / 1.8$

620 sensor (FLIR systems, CM3-U3-31S4M-CS) and a 1/1.8 lens with a 4.0-13mm variable focal

621 length (Tamron, M118VM413IRCS). We recorded 8-bit videos with a $75 \%$ M-JPEG

$622 \quad$ compression.

623 2) 30fps using a ELP 2.8-12mm Lens Varifocal Mini Box 1.3 megapixel USB Camera.

624 3) 50fps using a UCLA MiniCam 5 megapixel CMOS sensor (MT9P031I12STM-DP, ON

$625 \quad$ Semiconductor) 
627 Histology

628 Mice were transcardially perfused with phosphate-buffered saline (PBS) followed by $4 \%$

629 paraformaldehyde (PFA) in PBS. Brains were dissected, post-fixed in 4\% PFA for 12-24h and placed in

$63030 \%$ sucrose for 24-48 hours. They were then embedded in Optimum Cutting Temperature (OCT, Tissue

$631 \mathrm{Tek}$ ) and stored at $-80^{\circ} \mathrm{C}$ until sectioning. 60um floating sections were collected into PBS. Sections were

632 washed $3 \times 10 \mathrm{~min}$ in PBS and then blocked in $0.3 \%$ PBST containing 10\% normal donkey serum

633 (JacksonImmunoresearch, 17-000-121) for 2h. Sections were then stained with rabbit anti-RFP (Rockland

634 600-41-379 at 1:2000) in 0.3\% PBST containing 3\% donkey serum overnight at $4{ }^{\circ} \mathrm{C}$. The following day,

635 sections were washed $3 \times 5 \mathrm{~min}$ in PBS and then stained with secondary antibody (JacksonImmunoresearch

636 Cy3 donkey anti-rabbit $\operatorname{IgG}(\mathrm{H}+\mathrm{L}) 711-165-152,1: 1000)$ in $0.3 \%$ PBST containing 5\% donkey serum for

6372 hours at room temperature. Sections were then washed 5 min with PBS, 15 min with PBS+DAPI

638 (Thermofisher Scientific, D1306, 1:4000), and then 5 min with PBS. Sections were mounted on glass

639 slides using FluoroMount-G (ThermoFisher, 00-4958-02) and then imaged at 10x with a Leica slide

640 scanning microscope (VT1200S).

641

\section{Manual annotation of behavior}

643 Two-minute samples of each video recording were manually annotated by one-to-three highly trained

644 individuals for freezing behavior. One-minute intervals were chosen from the beginning and end of the

645 video recordings to capture diverse behaviors. In some cases, the entire video was annotated. Freezing

646 was defined as the absence of movement except for respiration. Video frames (PointGrey: 36,000 frames;

647 PointGrey+Opto: 36,000 frames; Webcam: 21,600 frames; MiniCAM: 46,069 frames; Rat videos: 40,320

648 frames; External mouse videos: 14,400 frames).

650 Statistical analyses

651 Statistical analyses were performed in MATLAB or GraphPad Prism. 


\section{Computer workstation specs}

654 We trained networks in DLC and analyzed videos using two different custom-built workstations (Intel

655 Core i9-9900K processor (8x 3.60GHz/16MB L3 Cache), 2x16GB DDR4-3000 RAM, NVIDIA GeForce

656 RTX 2070 SUPER - 8GB GDDR6; AMD RYZEN 9 3950x processor (16x3.5GHz/64MB L3 Cache),

657 16GB DDR4 RAM, Gigabyte GeForce RTX 2060 SUPER 8GB WINDFORCE OC). BehaviorDEPOT

658 can run on any personal computer and does not require a GPU.

659

\section{Installation of BehaviorDEPOT}

661 Detailed instructions on BehaviorDEPOT installation can be found on GitHub:

662 https://github.com/DeNardoLab/BehaviorDEPOT. Briefly, after installing a recent version of MATLAB

$663(2018+)$, BehaviorDEPOT can be downloaded from Github and installed with a single click as either a

664 MATLAB application or as a standalone exe file. Updates to the application will be added to the Github

665 repository as they come available. We welcome feedback and bug reports on the BehaviorDEPOT Github

666 page and encourage users to watch the page to be aware of any new releases.

667

668 MiniCAM Instructions and Installation Descriptions of fabrication and use of MinCAMs can be found 669 on GitHub: https://github.com/Aharoni-Lab/MiniCAM. 


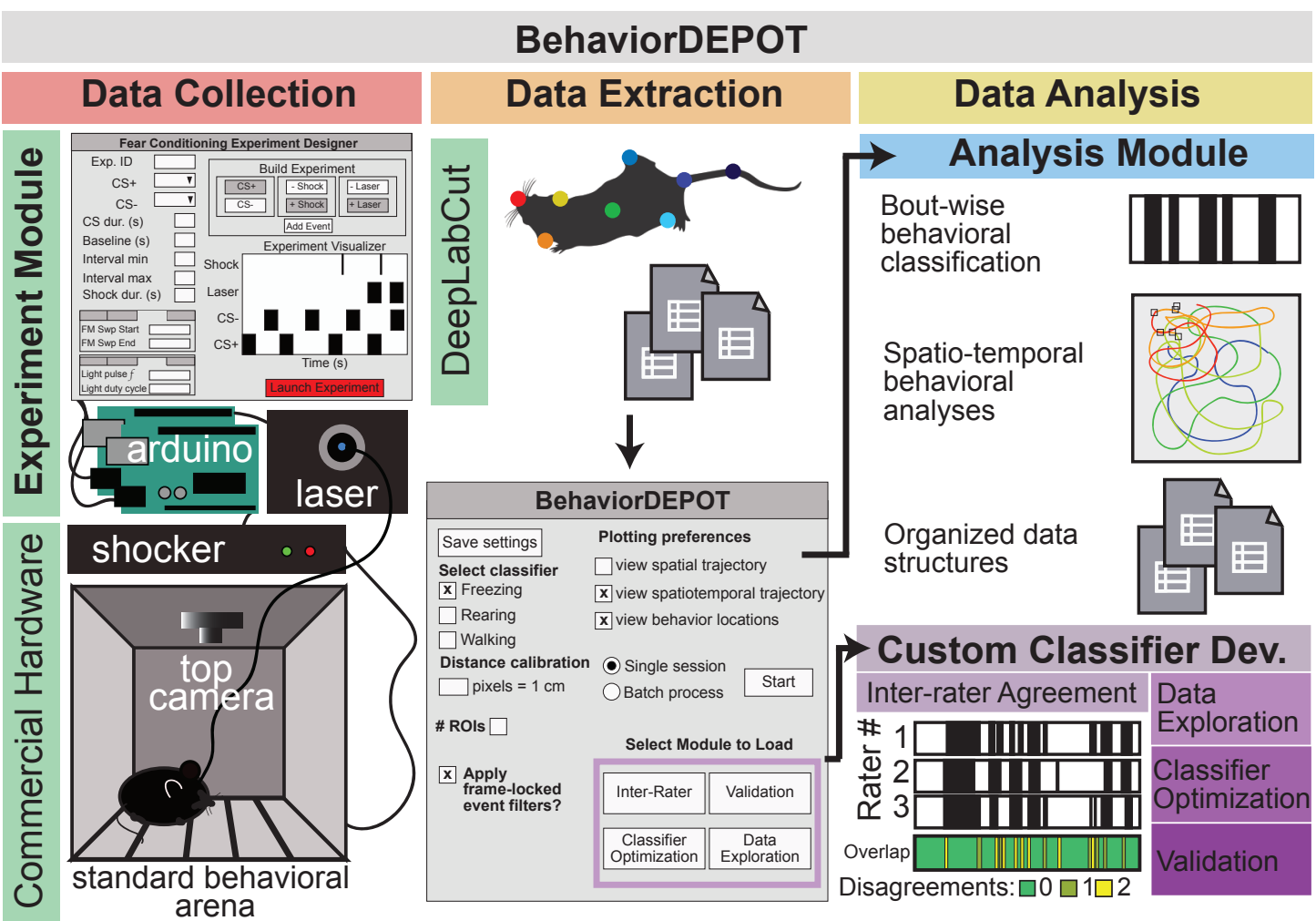

680 Figure 1. BehaviorDEPOT overview. The Experiment Module is a MATLAB GUI based application that 681 allows users to design and run fear conditioning experiments. The software uses Arduinos to interface 682 with commercially available hardware (e.g., shockers and lasers) to control stimuli. Behavior videos are acquired with webcams or machine-learning quality cameras. Video recordings are analyzed with pretrained DeepLabCut models. The video and pose estimates are the inputs for a second MATLAB GUI that controls the Analysis Module for customizable analyses of freezing, rearing, and moving. Four 


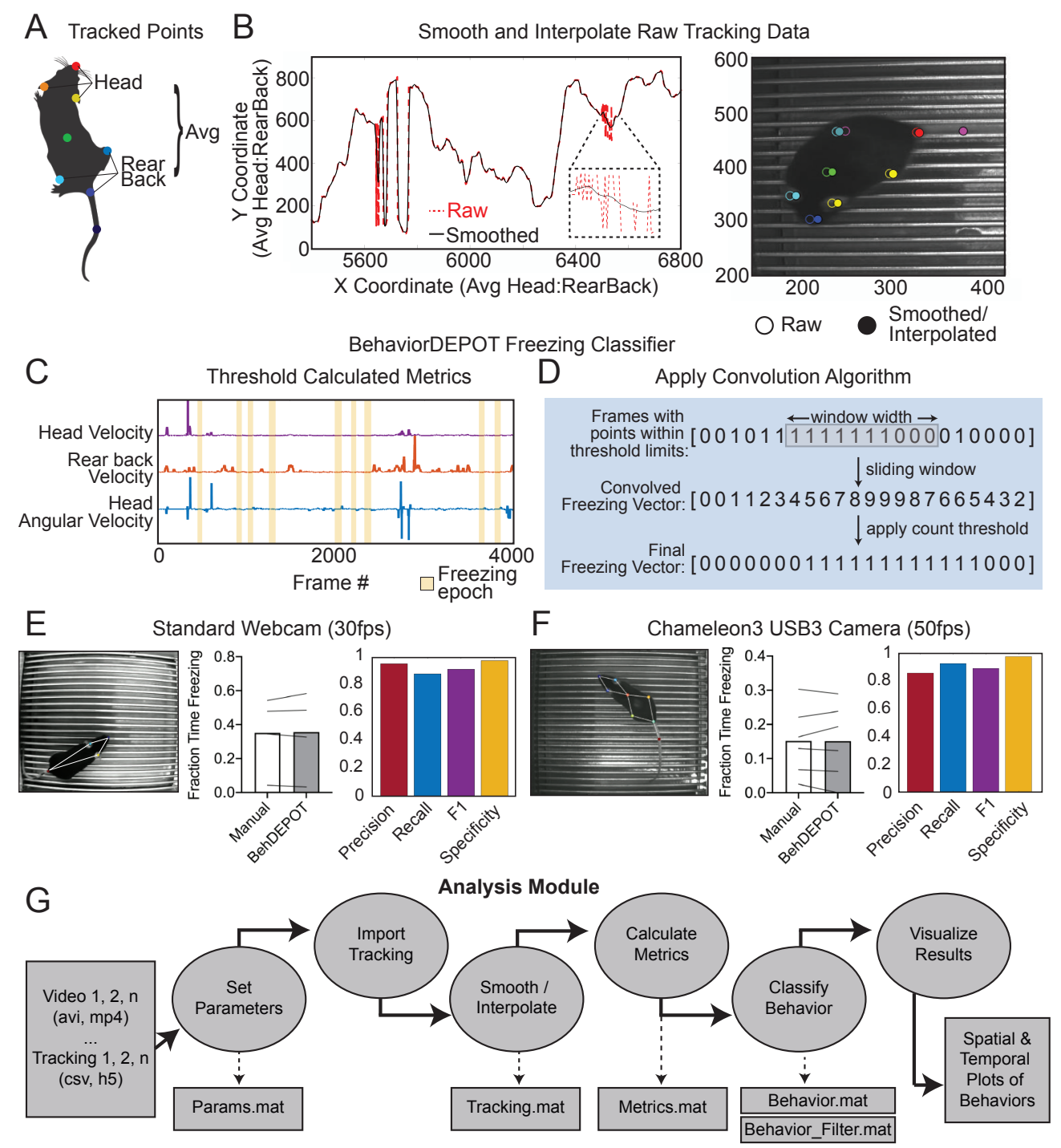

Figure 2. The Analysis Module. A. Metrics based on individual tracked points and weighted averages are calculated and stored in BehaviorDEPOT data matrices. B. Visualization of the effects of the LOWESS smoothing and interpolation algorithms for the weighted average of head and rear back (left) and for all tracked points in a representative example frame (right). C. Visualization of metrics that form the basis of the BehaviorDEPOT freezing classifier. Colored lines represent framewise calculated values for each metric. Yellow bars indicate freezing epochs. D. Visualization of the convolution algorithm employed by the BehaviorDEPOT freezing classifier. A sliding window of a specified width produces a convolved freezing vector in which each value represents the number of freezing frames visible in the window at a given frame. An adjustable count threshold converts the convolved freezing vector into the final binary freezing vector. E. Evaluation of freezing classifier performance on videos recorded at $30 \mathrm{fps}$ with a standard webcam (Precision: 0.95, Recall: 0.88, F1: 0.97, Specificity: 0.91). F. Evaluation of freezing classifier performance on videos recorded at $50 \mathrm{fps}$ with a machine learning-quality camera (Precision: 0.86, Recall: 0.92, F1: 0.89, Specificity: 0.97). G. The Analysis Module workflow. Videos and accompanying pose tracking data are the inputs. Pose tracking and behavioral data is vectorized and saved in MATLAB structures to facilitate subsequent analyses. 

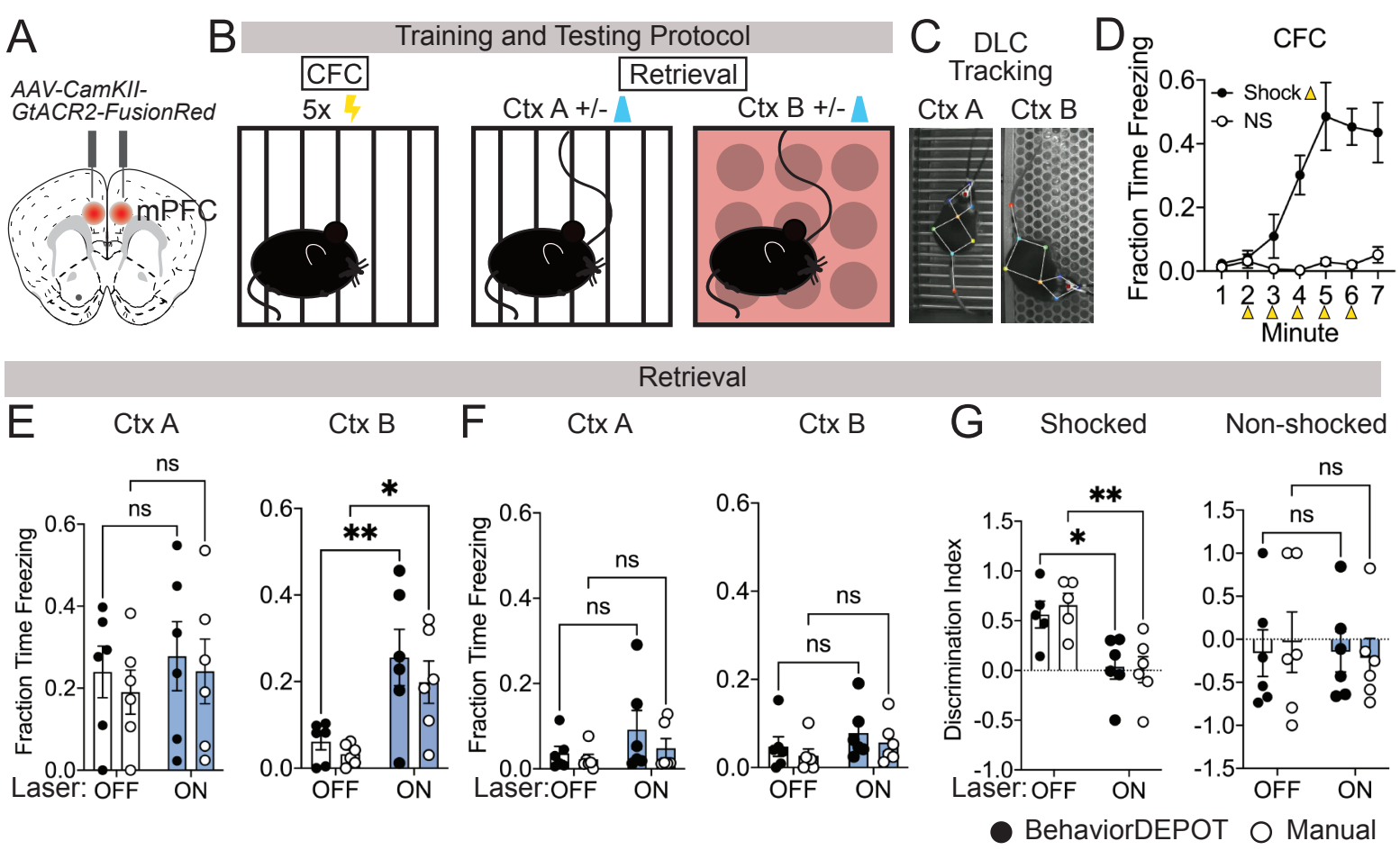

704

705

706

707

708

709

710

711

712

713

714

715

716

717

718

719

720

721

722

723
Figure 3. Use Case 1: Optogenetics. A. AAV1-CamKII-GtACR2-FusionRed was injected bilaterally into medial prefrontal cortex (mPFC). B. Behavioral protocol. Mice underwent contextual fear conditioning on day 1 . On day 2 , mice were returned to the conditioned context or a novel context in a counterbalanced fashion and received $2 \times 2 \mathrm{~min} 473 \mathrm{~nm}$ laser stimulation separated by 2 min laser off intervals. C. Example DLC tracking of mice attached to patch cords in different contexts. D. Quantification of contextual freezing during training analyzed with BehaviorDEPOT. E-F. Comparing human annotations to BehaviorDEPOT freezing classifier. E. Shocked mice: freezing in context A (left) and context B (right) with and without $\mathrm{mPFC}$ silencing $\left(\mathrm{CtxA}: F_{\text {laser }}(1,10)=0.42, P=0.53 ; F_{\text {rater }}(1,10)=0.35, P=0.57\right.$; CtxB: $F_{\text {laser }}(1,10)=26.51, P=0.0004 ; F_{\text {rater }}(1,10)=0.08, P=0.78$; Two-way repeated measures ANOVA, N=6 mice per group). F. Non-shocked controls: freezing in context A (left) and context B (right) with and without mPFC silencing $\left(F_{\text {laser }}(1,10)=3.60, P=0.09 ; F_{\text {rater }}(1,10)=0.79, P=0.39\right.$; Two-way repeated measures ANOVA, $\mathrm{N}=6$ mice per group). G. Discrimination index $=($ FreezeA - FreezeB $) /($ FreezeA + FreezeB $)$ for shocked mice $\left(F_{\text {laser }}(1,10)=17.54, P=0.002 ; F_{\text {rater }}(1,8)=0.09, P=0.77\right.$; Mixed-effects analysis, $\mathrm{N}=5-6$ per group) and non-shocked controls $\left(F_{\text {laser }}(1,10)=0.07, P=0.80 ; F_{\text {rater }}(1,8)=0.02, P=0.90\right.$; Two-way ANOVA). Error bars represent S.E.M. 


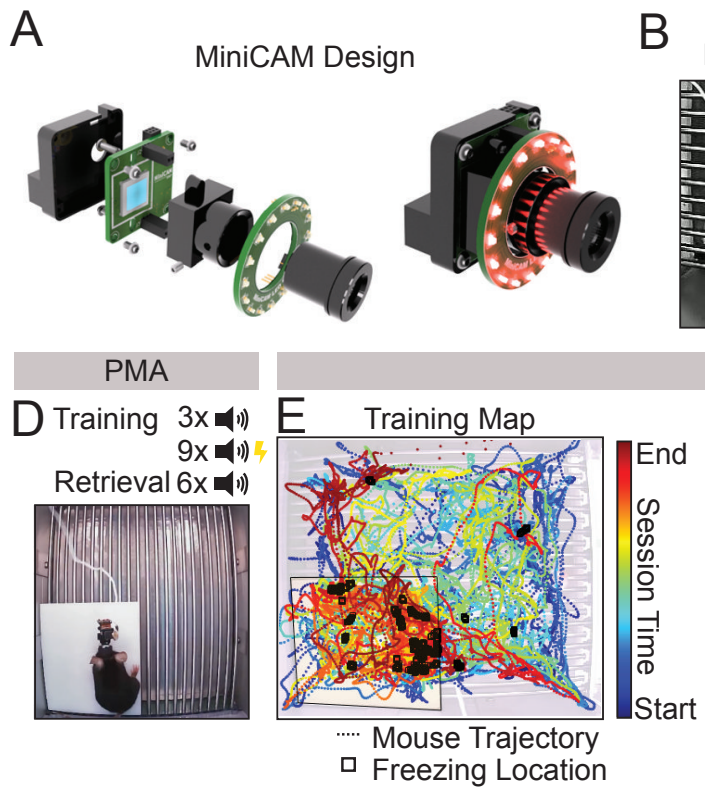

$\mathrm{H}$
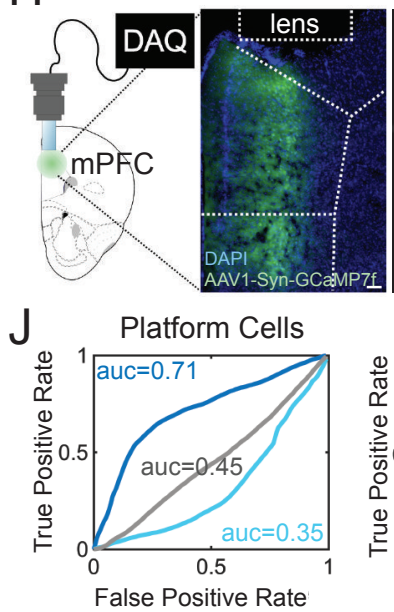
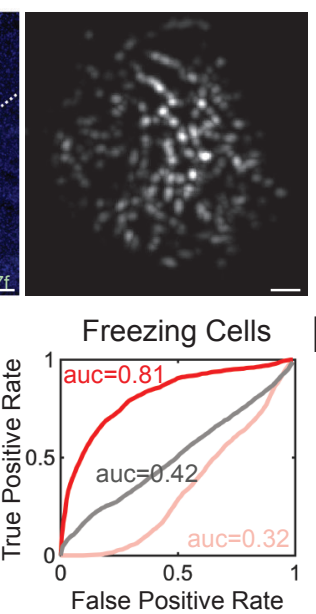
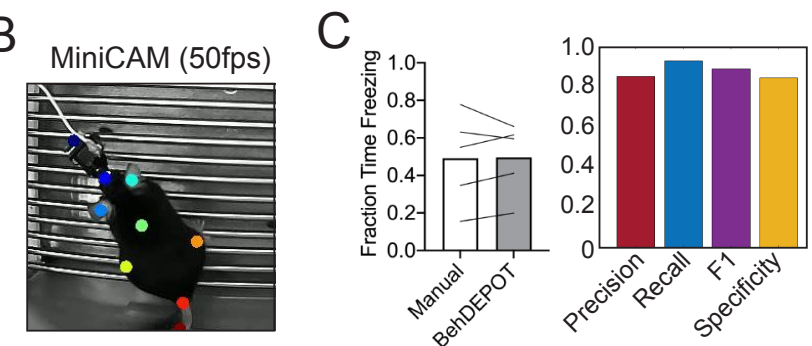

BehaviorDEPOT Output

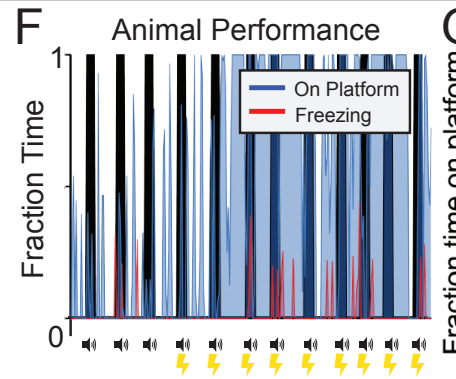

Tone \#

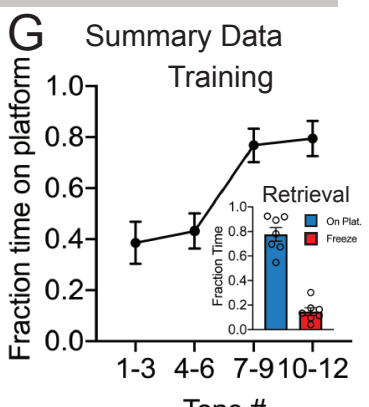

I
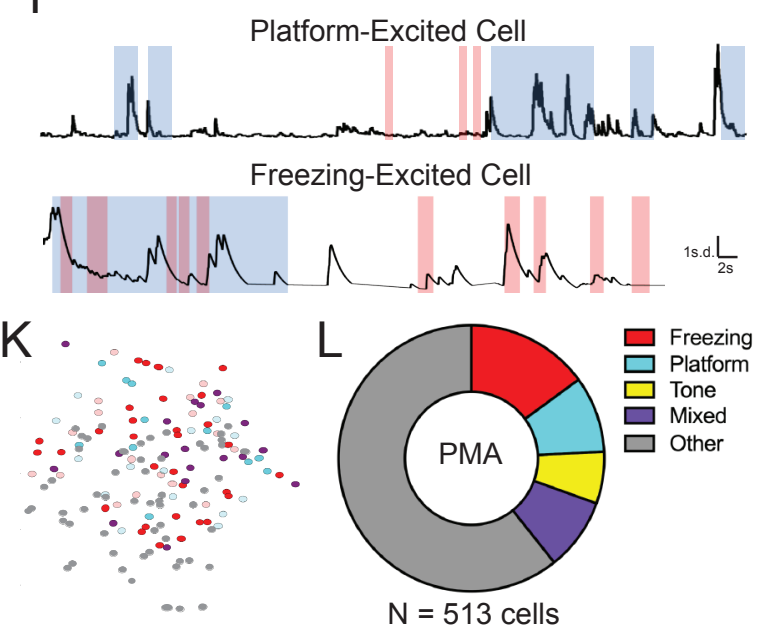

Figure 4. Use Case 2: Mice wearing Miniscopes. A. Design for MiniCAM, an open-source camera designed to interface with Miniscopes and pose tracking. B. Still frame from MiniCAM recording of mouse wearing a V4 Minscope. DLC tracked points are labeled with rainbow dots. C. Performance of freezing classifier on videos of mouse wearing Miniscope recorded with MiniCAM (Precision: 0.85; Recall: 0.93; F1 Score: 0.89; Specificity: 0.84). D. Task design. E. Sample BehaviorDEPOT output for mouse wearing Miniscope during PMA. Map displays animal position over time as well as freezing locations (black squares). F. Temporal alignment of time on the platform (blue), time freezing (black), and tones. G. Summary data for training and retrieval. H. GCaMP7-exressing mPFC neurons imaged through a V4 Miniscope. I. Example $\mathrm{Ca}^{2+}$ traces from platform (top) and tone (bottom) modulated cells during time on the platform (blue) or time freezing (pink). J. Receiver operating characteristic (ROC) curves that were calculated for platform-modulated cells (excited cell: auc-0.71; suppressed cell: auc $=0.35$, unmodulated cell: auc $=0.45$ ) and freezing-modulated cells (excited cell: auc $=0.81$; suppressed cell: auc $=0.32$; unmodulated cell: auc $=0.42$ ). K. Example field of view showing locations of freezing- and platform-modulated $\mathrm{mPFC}$ neurons. L. Proportion of modulated cells of each functional type from 513 cells recorded across 3 mice. Scale bars, 100um. Error bars represent S.E.M. 
$740 \quad$ A

741

742

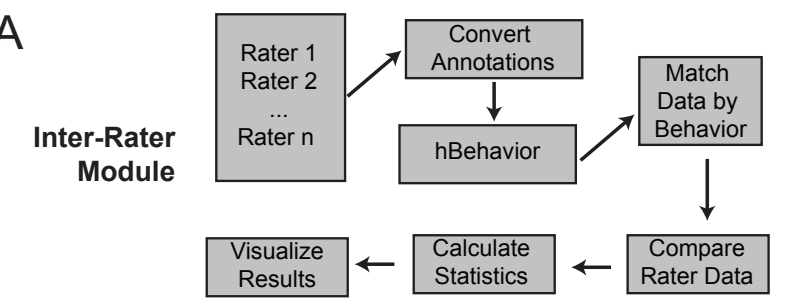

744

B

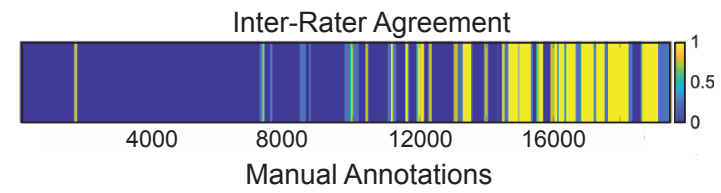

753 Figure 5. Inter-Rater Module. A. The Inter-Rater Module workflow. B. The Inter-Rater Module produces visualizations of framewise agreement levels (top) based on manual annotations from multiple human 
A

Data Exploration Module
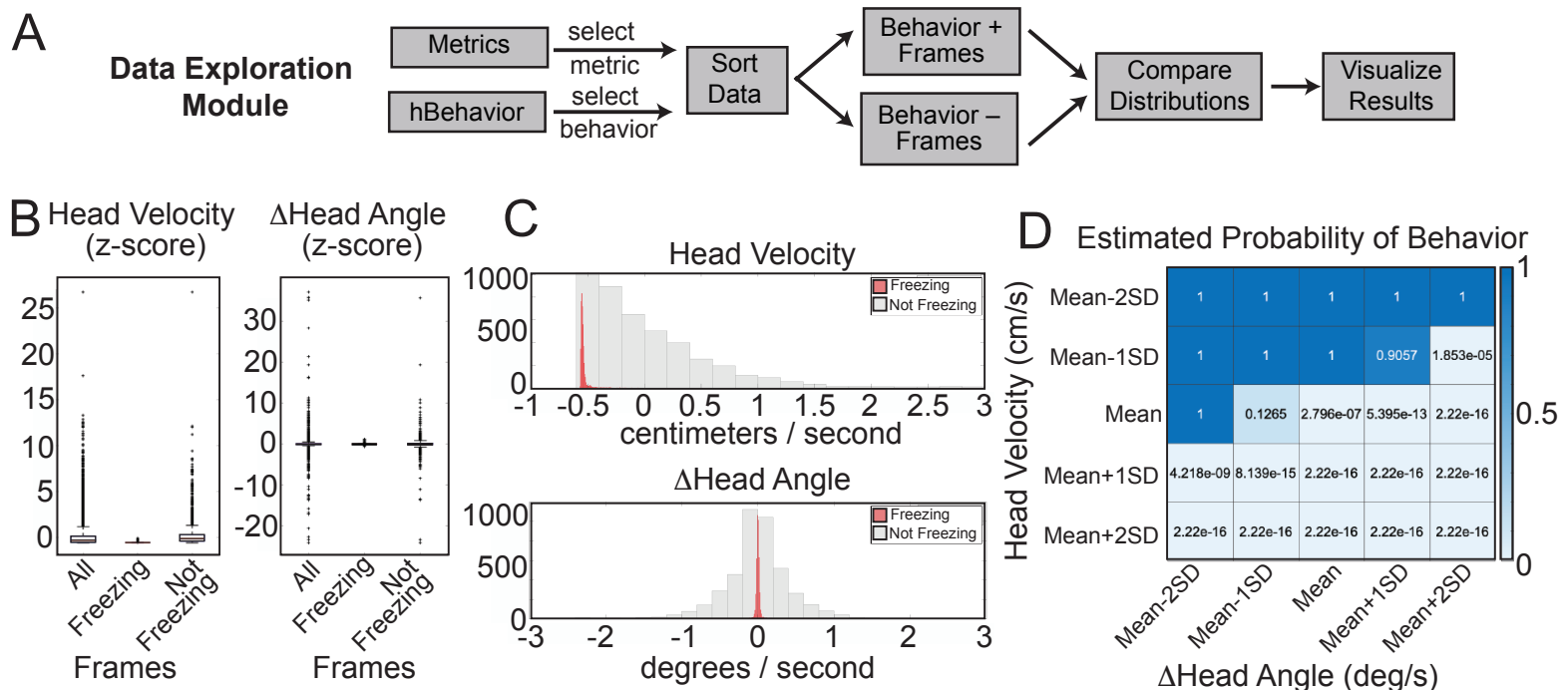
the Analysis Module and the human annotations of behavior. It sorts the data in a framewise manner, separating frames containing the behavior of interest from those without and then visualizes and compares the distribution of values for a metric of interest. B. Distributions of Z-scored values for head velocity (left) and change in head angle (right) are distinct for freezing vs. not freezing frames. Box plots represent median, $25^{\text {th }}$, and $75^{\text {th }}$ percentile. Error bars extend to the most extreme point that is not an outlier C. Histograms showing distribution of values for head velocity (top) and change in head angle (bottom) for freezing (red) vs. not-freezing (grey) frames. D. A generalized linear model (GLM) computes the predictive power of given metrics for frames containing the behavior of interest. 

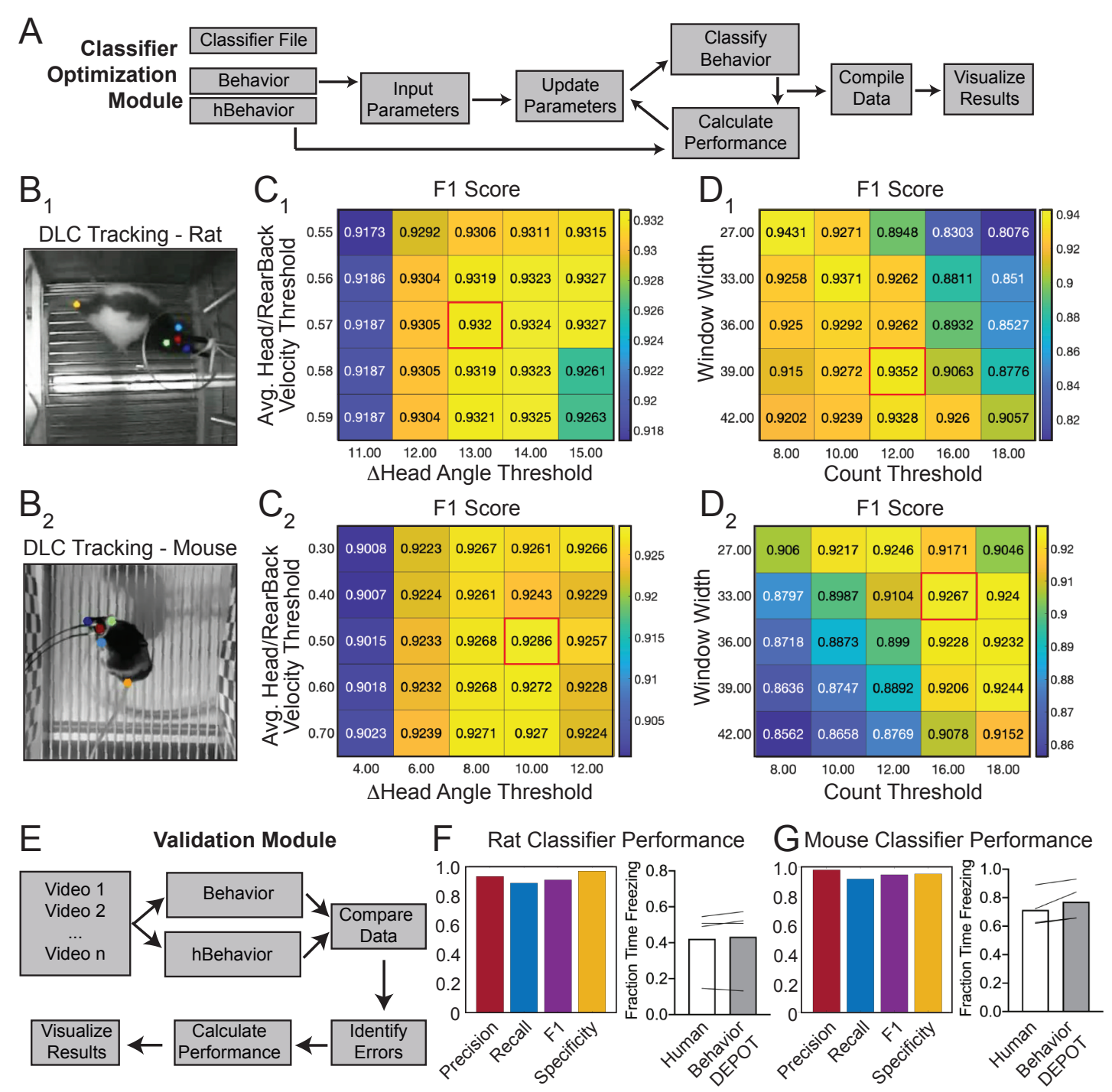

Figure 7. Use Case 3: Analysis of External Data using Classifier Optimization and Validation Modules. A. Classifier Optimization Module workflow. This module sweeps through a range of thresholds for statistics calculated based on tracked points and then compares the resulting behavioral classification to human annotations. $\mathrm{B}_{1,2}$. We optimized our freezing classifier for analysis of rat and mouse freezing in videos obtained from other laboratories. $\mathrm{C}_{1,2}$. The Classifier Optimization Module iteratively sweeps through a range of thresholds for different metrics and reports F1 scores. The module first swept through a range of values for head/rear back velocity and change in head angle. $\mathrm{D}_{1,2}$. The highest $\mathrm{F} 1$ score (red box) was selected and then a subsequent sweep through two additional value ranges (for window width and count threshold from the smoothing algorithm) produced an equivalent or higher F1 score (red box). E. Once the user has identified candidate threshold values, the Validation Module reports recall, precision, F1, and specificity scores. F. The BehaviorDEPOT classifier performed robustly on videos of rats recorded in a different lab (Precision $=0.93$; Recall $=0.88 ; \mathrm{F} 1=0.91$; Specificity $=0.96$ ). videos, $P=0.89$, Mann-Whitney $\mathrm{U})$. G. The BehaviorDEPOT classifier performed robustly on videos of mice recorded in a different lab (Precision $=0.98$; Recall $=0.92 ; \mathrm{F} 1=0.95$; Specificity $=0.95$ ). 

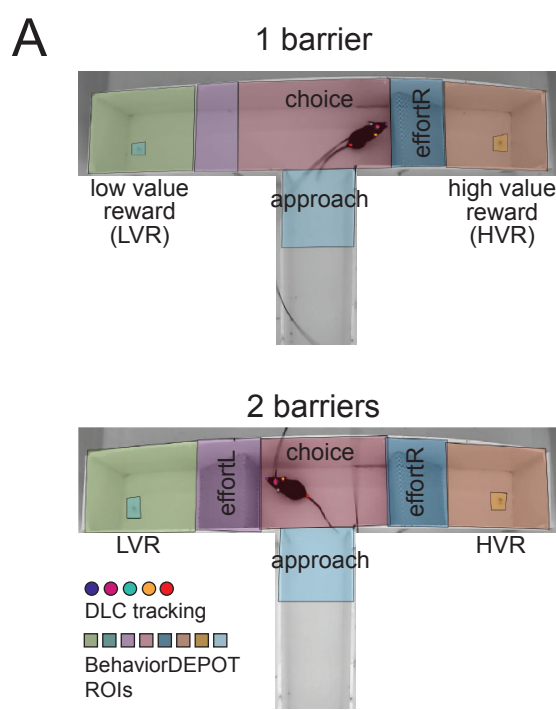

D

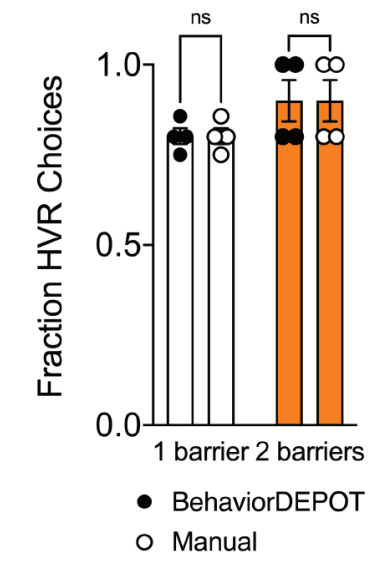

B Pose Tracking

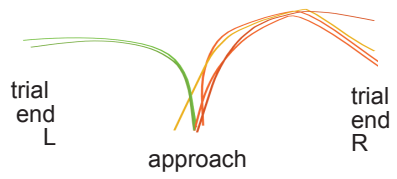

- Mouse trajectory, left choice - Mouse trajectory, right choice $>$ VTE trial
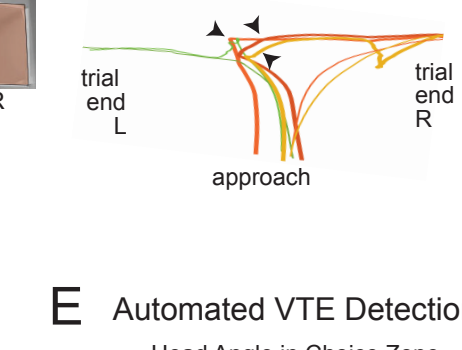

E Automated VTE Detection
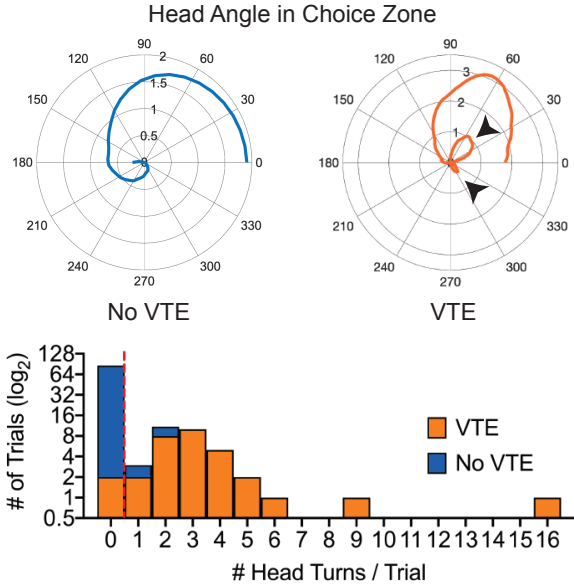

C Automated Trial Detection

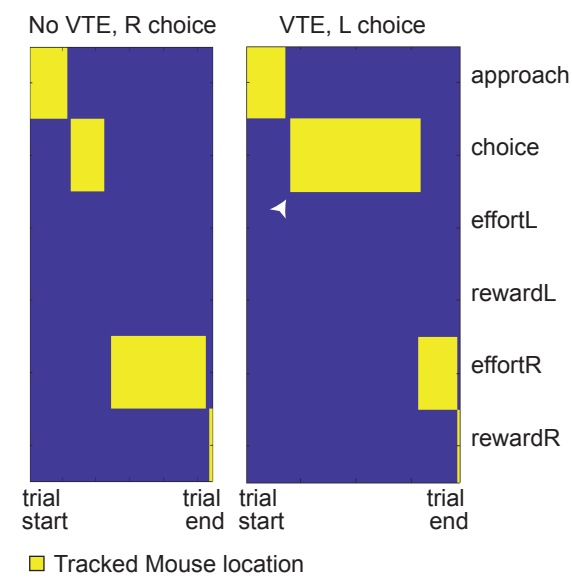

$$
\text { F } \quad \text { VTE Classifier }
$$

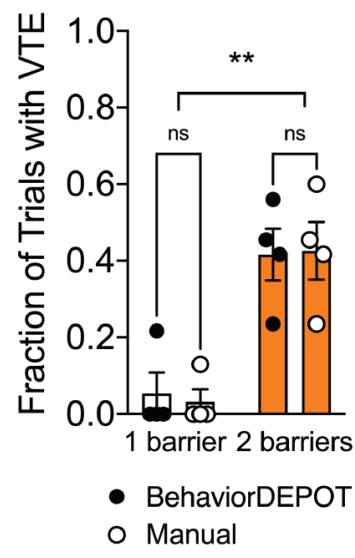

Figure 8. Use Case 4: Automated analysis of an effort-based decision-making T-maze task A. Screen shots showing DLC tracking in a 1-barrier (top) and 2-barrier (bottom) T-maze and ROIs used for analysis in BehaviorDEPOT. B. Sample mouse trajectories in a 1_barrier (left) and 2-barrier (right) Tmaze. Lines represent individual trials for 1 mouse. Blue lines represent right choices, orange lines represent left choices, and thick lines indicate vicarious trial and error (VTE). C. Illustration of automated trial definitions. D. Automated choice detection using BehaviorDEPOT. BehaviorDEPOT indicated choice with $100 \%$ accuracy $\left(F_{\text {Rater }}(1,6)=6.84, P>0.99, F_{\text {Barriers }}(1,7)=4.02, P=0.09 ; F_{\text {Subject }}(6,7)=0.42\right.$, $P=0.84$, 2-way ANOVA with Sidak post-hoc comparisons, 84 trials, $\mathrm{N}=4$ mice). E. Top: Polar plots show representative head angle trajectories when the mouse was in the choice zone during a trial without VTE (left) and with VTE (right). Bottom: Histogram of head turns per trial for trials without VTE (blue) and with VTE (orange). Red dotted line indicates selected threshold. F. Fraction of trials with VTE during 1barrier and 2-barrier sessions, comparing manual annotations to BehaviorDEPOT classification $\left(F_{\text {RaterxBarriers }}(1,6)=0.04, P=0.85, F_{\text {Rater }}(1,7)=0.03, P=0.85 ; F_{\text {Barriers }}(1,6)=22.9, P=0.003,2\right.$-way ANOVA with Sidak post-hoc comparisons, 102 trials, $\mathrm{N}=4$ mice). Error bars represent S.E.M. 


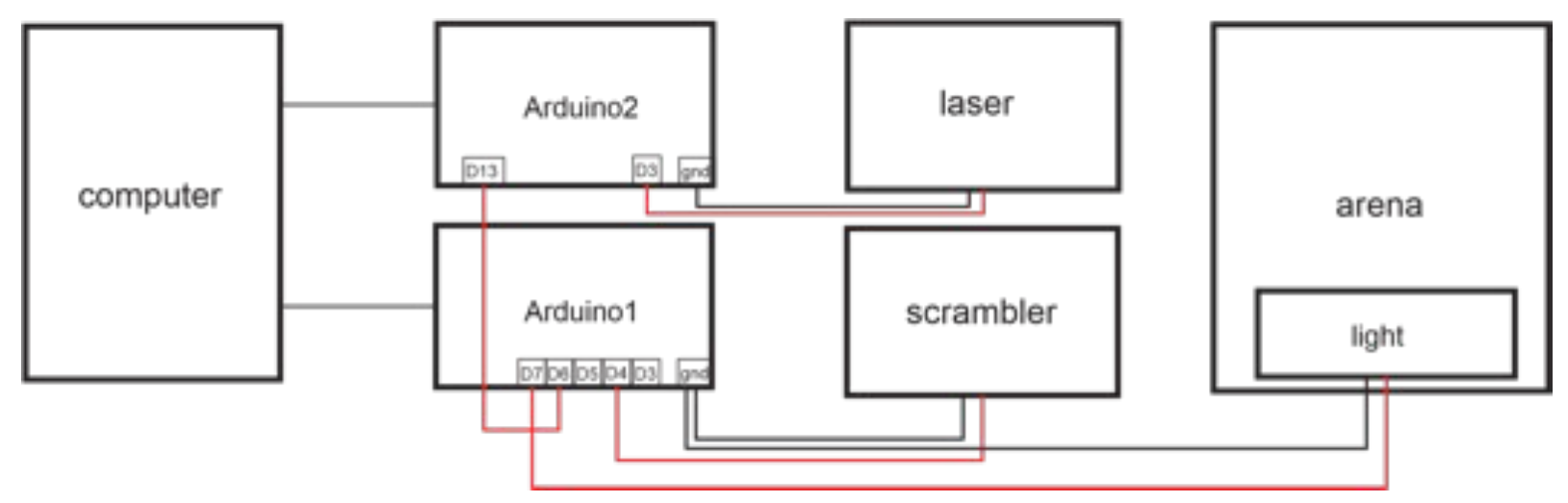

825 Supplemental Figure 1. Example arrangement of Arduino interface between computer, fear conditioning 826 and optogenetics hardware. The Experiment Module controls two Arduinos that control delivery of the 827 scrambled shocker, and a light (for use as a conditioned cue), and the laser for optogenetics, respectively. 828 MATLAB software triggers the conditioned tone.

829

830 
A
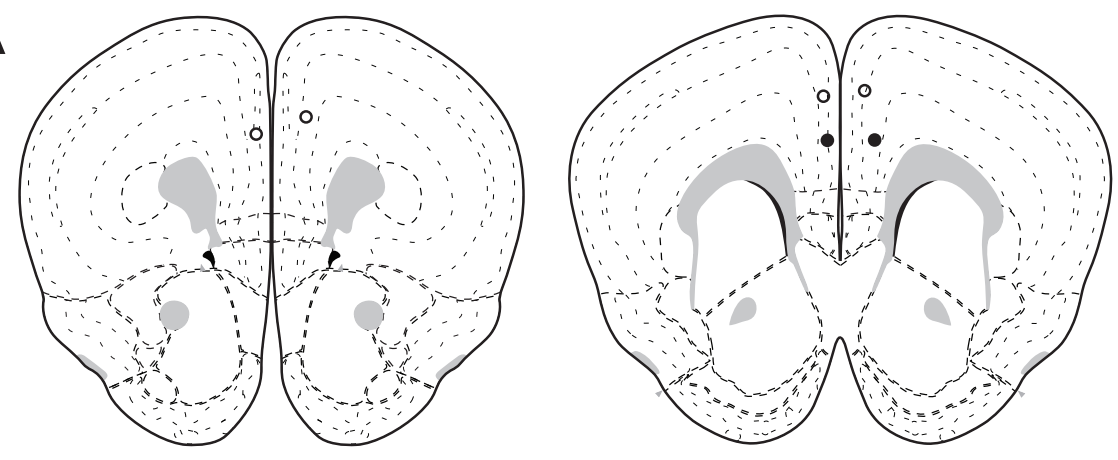

838
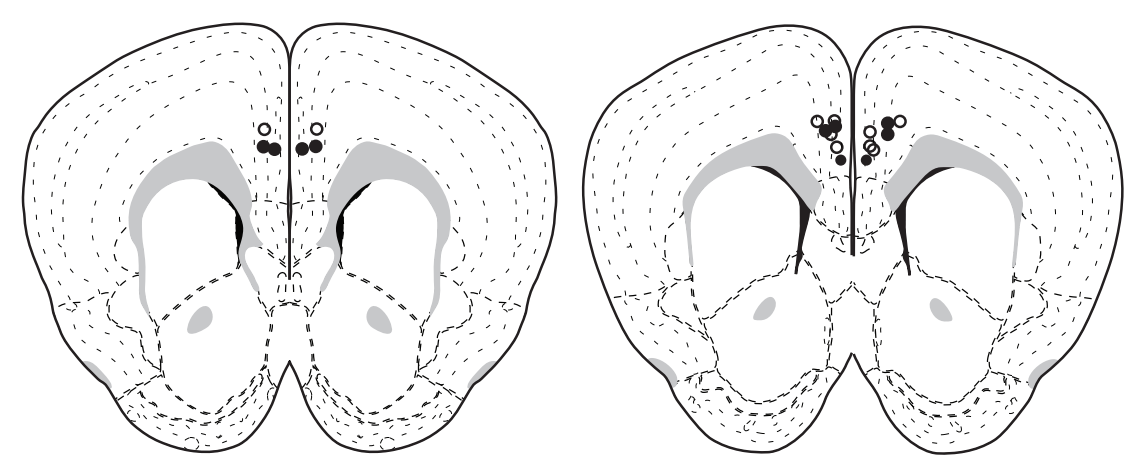

Shocked

Non-Shocked
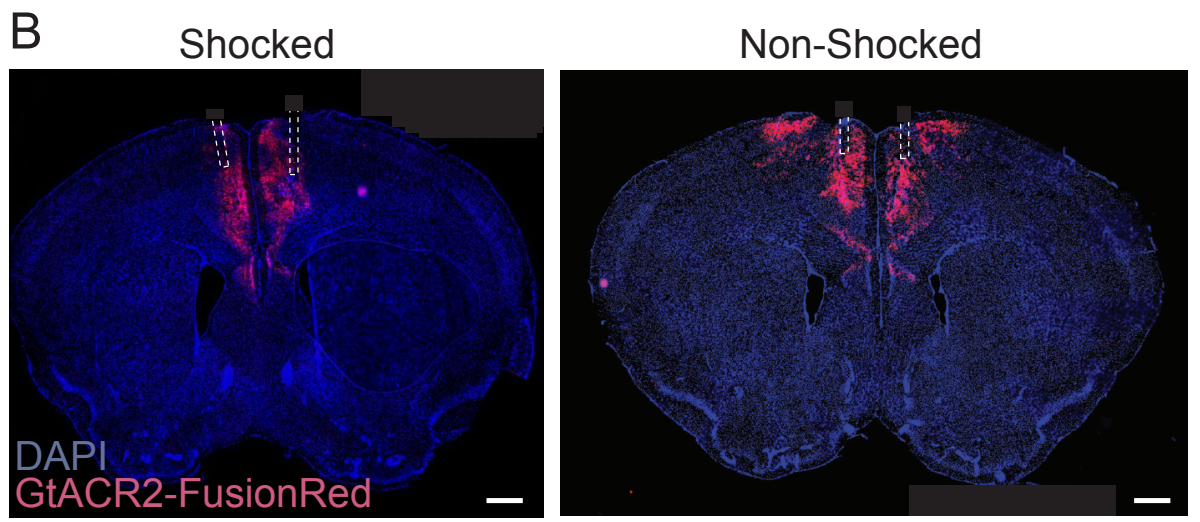

Supplemental Figure 2. A. Optic fiber cannula placements for experiment described in Figure 3. B.

840 stGtACR2 -FusionRed expression and bilateral fiber placement for representative shocked and non- 